\title{
UPDATE ON THE STATUS OF HADRONIC SQUEEZED CORRELATIONS AT RHIC ENERGIES
}

\author{
S. S. Padula ${ }^{a}$, D. M. Dudek ${ }^{a}$, O. Socolowski, Jr. ${ }^{b}$ \\ ${ }^{a}$ Instituto de Física Teórica — UNESP, São Paulo, SP, Brazil \\ ${ }^{b}$ IMEF-FURG, Rio Grande, RS, Brazil
}

\begin{abstract}
In high-energy heavy-ion collisions, a hot and dense medium is formed, where the hadronic masses may be shifted from their asymptotic values. If this mass modification occurs, squeezed back-to-back correlations (BBC) of particle-antiparticle pairs are predicted to appear, both in the fermionic (fBBC) and in the bosonic (bBBC) sectors. Although they have unlimited intensity even for finite-size expanding systems, these hadronic squeezed correlations are very sensitive to their time emission distribution. Here we discuss results in case this time emission is parameterized by a Lévy-type distribution, showing that it reduces the signal even more dramatically than a Lorentzian distribution, which already reduces the intensity of the effect by orders of magnitude, as compared to the sudden emission. However, we show that the signal could still survive if the duration of the process is short, and if the effect is searched for lighter mesons, such as kaons. We compare some of our results to recent PHENIX preliminary data on squeezed correlations of $K^{+} K^{-}$pairs.
\end{abstract}

PACS: $25.75 .-\mathrm{q}$

The history of the squeezed correlations between particle-antiparticle pairs started in the early 1990s [1], but its final formulation was proposed almost a decade later, by M. Asakawa et al. [2]. In such an approach, the squeezed back-to-back correlations (BBC) of bosonantiboson pairs resulted from a quantum mechanical unitary transformation relating in-medium quasiparticles to two-mode squeezed states of their free counterparts, originated in a modification of the particles' mass in the hot and dense medium. Shortly after that, P. K. Panda et al. [3] showed that a similar BBC between fermion-antifermion pairs should exist, if the masses of these particles were modified in-medium. Both the fermionic (fBBC) and the bosonic (bBBC) back-to-back squeezed correlations are described by analogous formalisms, being both positive correlations of unlimited intensity. In the remainder of this paper, we focus our discussion on the case of charged kaons, i.e., $K^{+}$and $K^{-}$pairs, for which the squeezed correlations are predicted. Since in this case there is no contribution from identical particles, the correlation function is written as $C_{s}\left(\mathbf{k}_{1}, \mathbf{k}_{2}\right)=1+\frac{\left|G_{s}(1,2)\right|^{2}}{G_{c}(1,1) G_{c}(2,2)}$, where $G_{c}(i, i)=\omega_{\mathbf{k}_{i}}\left\langle\hat{a}_{\mathbf{k}_{i}}^{\dagger} \hat{a}_{\mathbf{k}_{i}}\right\rangle=\omega_{\mathbf{k}_{i}} d^{3} N / d \mathbf{k}_{i}$ is the spectrum of each particle $(i=1,2)$ and $G_{s}(1,2)=\sqrt{\omega_{\mathbf{k}_{1}} \omega_{\mathbf{k}_{2}}}\left\langle\hat{a}_{\mathbf{k}_{1}} \hat{a}_{\mathbf{k}_{2}}\right\rangle$ is the squeezed amplitude, which gives a nonzero contribution if the hadronic masses are modified in-medium. This nonvanishing contribution to $\left\langle\hat{a}_{\mathbf{k}_{1}}^{(\dagger)} \hat{a}_{\mathbf{k}_{2}}^{(\dagger)}\right\rangle$ is originated in the Bogolyubov-Valatin transformation relating the asymptotic operators, $\hat{a}_{k}$ $\left(\hat{a}_{k}^{\dagger}\right)$, to their in-medium counterparts, $\hat{b}_{k}\left(\hat{b}_{k}^{\dagger}\right)$, i.e., $a_{k}=c_{k} b_{k}+s_{-k}^{*} b_{-k}^{\dagger} ; a_{k}^{\dagger}=c_{k}^{*} b_{k}^{\dagger}+s_{-k} b_{-k}$, where $c_{k}=\cosh \left(f_{k}\right), s_{k}=\sinh \left(f_{k}\right)$. The argument, $f_{i, j}(x)=\frac{1}{2} \log \left[\frac{K_{i, j}^{\mu}(x) u_{\mu}(x)}{K_{i, j}^{* \nu}(x) u_{\nu}(x)}\right]$, is 
the squeezing parameter, where $K_{i, j}^{\mu}(x)=(1 / 2)\left(k_{i}^{\mu}+k_{j}^{\mu}\right)$ is the average of the momenta of each particle, and $u_{\mu}$ is the flow velocity of the system. The details of the formalism and relations used to produce the results shown here were derived in $[2,4-6]$.

In $[2,3]$, the case of static infinite medium was considered and later, in [4-6], a finite system expanding with moderate radial flow was analyzed. For simplicity, the expansion was considered to be nonrelativistic and the squeezing parameter, to be flow and momentumindependent. A constant mass shift was assumed, homogeneously distributed over the system or in part of it, and linearly related to the asymptotic mass, i.e., $m_{*}=m \pm \delta M$ [3-6]. These simplifications allowed one to obtain analytical expressions for the BBC correlation functions, whose forms were detailed in [4-6]. Several interesting features of the squeezed correlations were discussed in those references. One of them, in particular, is rather striking; i.e., the time emission distributions play a crucial role in the possible observation of the squeezing correlations. In previous works, it was shown that the strength of the squeezed correlation could be very high if the emission was instantaneous, i.e., described by a $\delta$ function at freezeout. In the case of $K^{+} K^{-}$pairs this could reach values as high as $C(k,-k) \sim 330$, at the maximal mass shift, $m_{*}=350 \mathrm{MeV}$, for expanding systems with radial flow velocity, $\langle u\rangle=0.5$, as illustrated in Fig. 1, $a$. However, if the emission lasts a finite interval, the
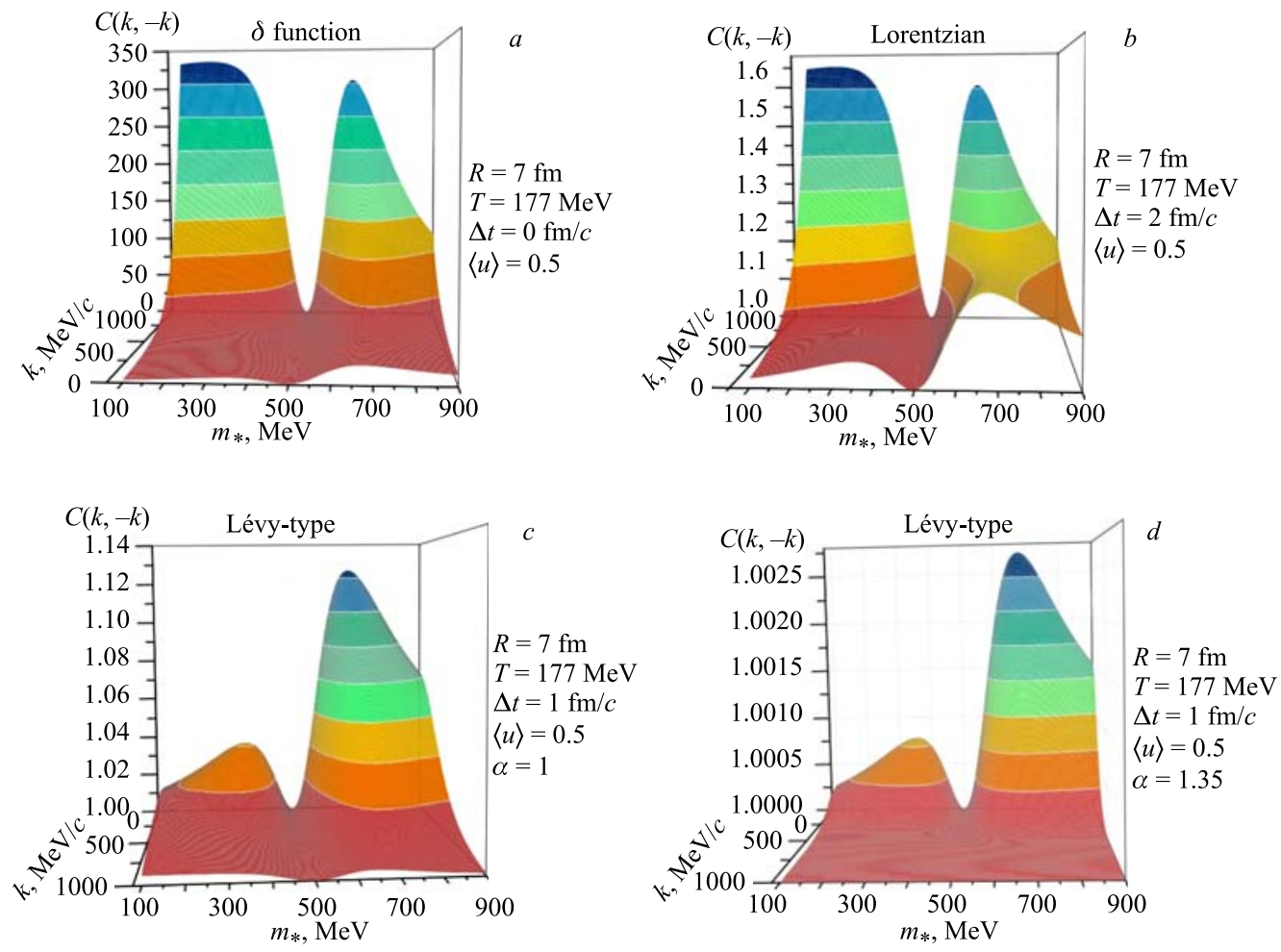

Fig. 1. The squeezed to correlation function in terms of the modified mass, $m_{*}$, and the relative momentum of the pair, $k=|\mathbf{k}|$, shown for different time distributions. a) Results for the case of a $\delta$ function in time. $b$ ) Results for a Lorentzian with $\Delta t=2 \mathrm{fm} / c ; c$ ) for a Lévy distribution with $\alpha=1$, and $d$ ) for $\alpha=1.35$, both for $\Delta t=1 \mathrm{fm} / c$ 
squeezed term should be multiplied by a factor which may reduce this intensity considerably. For instance, for a Lorentzian time emission, $|\mathcal{F}(\Delta t)|^{2}=\left[1+\left(\omega_{1}+\omega_{2}\right)^{2} \Delta t^{2}\right]^{-1}$, with a time interval $\Delta t=2 \mathrm{fm} / c$, as adopted in [2-6], the maximum intensity of the squeezed correlation function may be roughly a factor of 20 smaller than in the case of instantaneous emission. This is shown in Fig. 1, $b$. Nevertheless, since we do not know a priori which functional form would be privileged by nature, we also investigate the time emission as a symmetric, $\alpha$-stable Lévy distribution, $|F(\Delta t)|^{2}=\exp \left\{-\left[\Delta t\left(\omega_{1}+\omega_{2}\right)\right]^{\alpha}\right\}$. This functional form was used to fit PHENIX data [7] on two- and three-particle Bose-Einstein correlations. The distribution index was found to be $\alpha \sim 1$, for $0.2<m_{T}<0.3 \mathrm{GeV}$, and $\alpha \sim 1.35$, for $0.3<p_{T}<0.45 \mathrm{GeV} / c$, for which we investigate the time emission factor. This is shown in Fig. 1, $c$ and $d$, respectively. We see that the Lévy distribution reduces the intensity of the squeezed correlation function even more dramatically than the Lorentzian distribution.

The results shown in Fig. 1 are important but not practical for the purpose of searching for hadronic squeezed correlations empirically, since the modified mass, $m_{*}$, is not observable outside the hot and dense medium, and the particle and antiparticle momenta are hardly measured as exactly back-to-back. Instead, we should consider distinct values for the momenta of the particles, $\mathbf{k}_{1}$ and $\mathbf{k}_{2}$, and combine them in measurable quantities, e.g., as their average, $\mathbf{K}_{12}=(1 / 2)\left(\mathbf{k}_{1}+\mathbf{k}_{2}\right)$, and their relative momenta, $\mathbf{q}_{12}=\left(\mathbf{k}_{1}-\mathbf{k}_{2}\right)$ [4-6]. This approach considers nonrelativistic momenta and therefore has its application constrained to this limit. For a relativistic treatment, see discussion in [8]. Naturally, the values of the squeezed correlation function in Fig. 1 show only the maximum expected value, i.e., its intercept at $K_{12}=0$, for different values of $\left|\mathbf{q}_{12}\right|=\left|\mathbf{k}_{1}-\mathbf{k}_{2}\right|=|\mathbf{k}-(-\mathbf{k})|=2|\mathbf{k}|$.

In our nonrelativistic model we consider that the system formed in high-energy collisions is described by a Gaussian source with circular cross-sectional area of radius $R$ (see [4] for details). In the studies shown in Fig. 1 , the mass modification was supposed to be homogeneously distributed over all the system of radius $R=7 \mathrm{fm}$. However, the signal of the resulting correlation function falls too fast to unity if compared to the PHENIX preliminary data on squeezed hadronic correlations, presented in [9]. In contrast, we consider in Fig. 2, $a$ the result of the simulation with our model, for a small system with $R=1.5 \mathrm{fm}$, where the squeezed correlation is shown as a function of $2 K_{12}$, for particles with transverse momenta in the range $0.6<m_{T}<0.8$, as in the experiment. Results for several values of the modified mass, $m_{*}$, are shown. Although describing the trend of data reasonably well, specially for $m_{*}=460 \mathrm{MeV}$ (continuous line), such a radius is too small as compared with measurements from the PHENIX collaboration in similar conditions (see discussion in [5]). Besides, also the hypothesis of mass modification over all the system may be too strong. So, we consider also the two-volume case discussed in [4], in which the squeezing is assumed to be restricted to a smaller portion, with radius $R_{s}$, inside of the system of radius $R$. This is shown in Fig. 2, $b$ for several combinations of these values of radii, fixing the shifted kaon mass as $m_{*}=350 \mathrm{MeV}$. We see that some of the parameters in Fig. 2, $b$ describe the data qualitatively well, in particular the histograms corresponding to $R=7.0 \mathrm{fm}, R_{s}=5 \mathrm{fm}$ and $R=3.2 \mathrm{fm}$, $R_{s}=2 \mathrm{fm}$; i.e., there is ambiguity in the scenarios. Nevertheless, in case the statistics could be increased considerably, a study of the correlation function, $C\left(K_{12}, q_{12}\right)$, in terms of these variables in the plane $\left(2 K_{12}, q_{12}\right)$, could improve the distinction of different scenarios, as shown in Fig. 3, $a$ and $b$, for the most prominent sets of variables of Fig. 2, $b$.

As a final remark, we could say that a comparison of our two-volume nonrelativistic model with the preliminary PHENIX data on the squeezed hadronic correlations of [9] does 
$K^{+} K^{-}$squeezed correlations
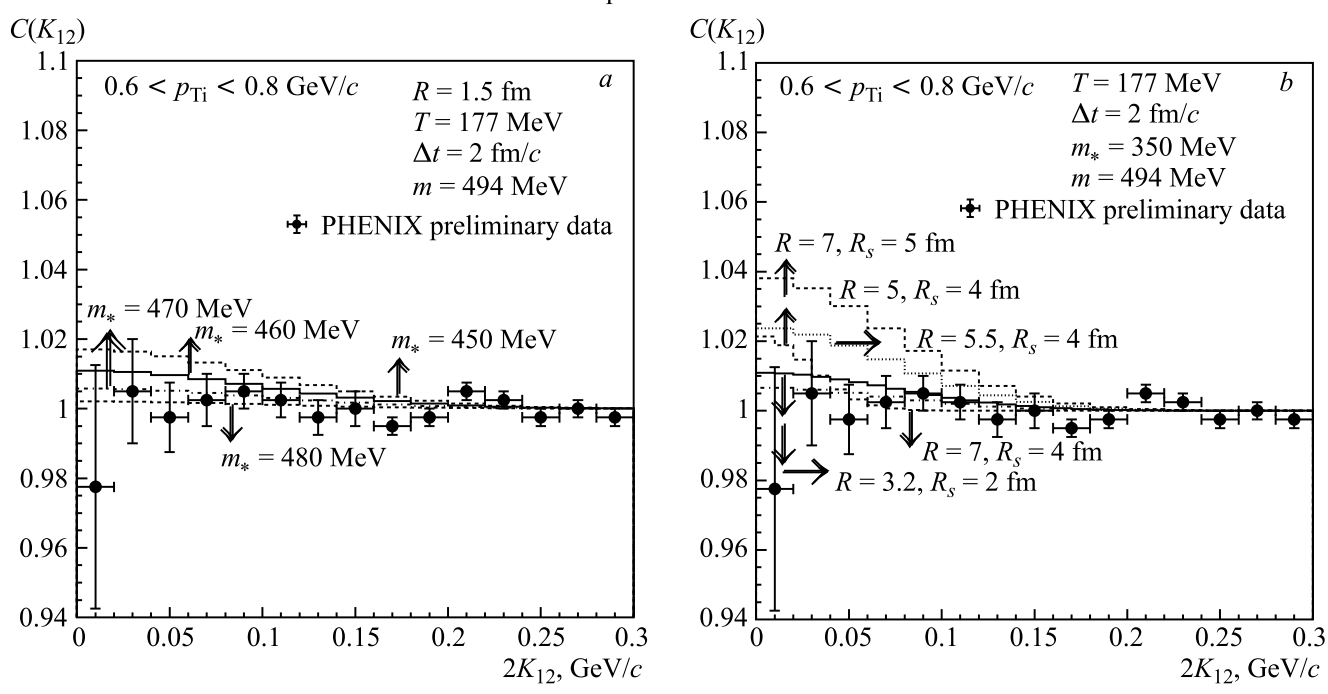

Fig. 2. a) The comparison with data of the squeezed correlation function versus $2 K_{12}$, for several values of $m_{*}$ and $R=1.5 \mathrm{fm} . b$ ) Similar results considering that the squeezing occurs in a smaller portion (with radius $R_{s}$ ) of the system of radius $R$, for different values of radii

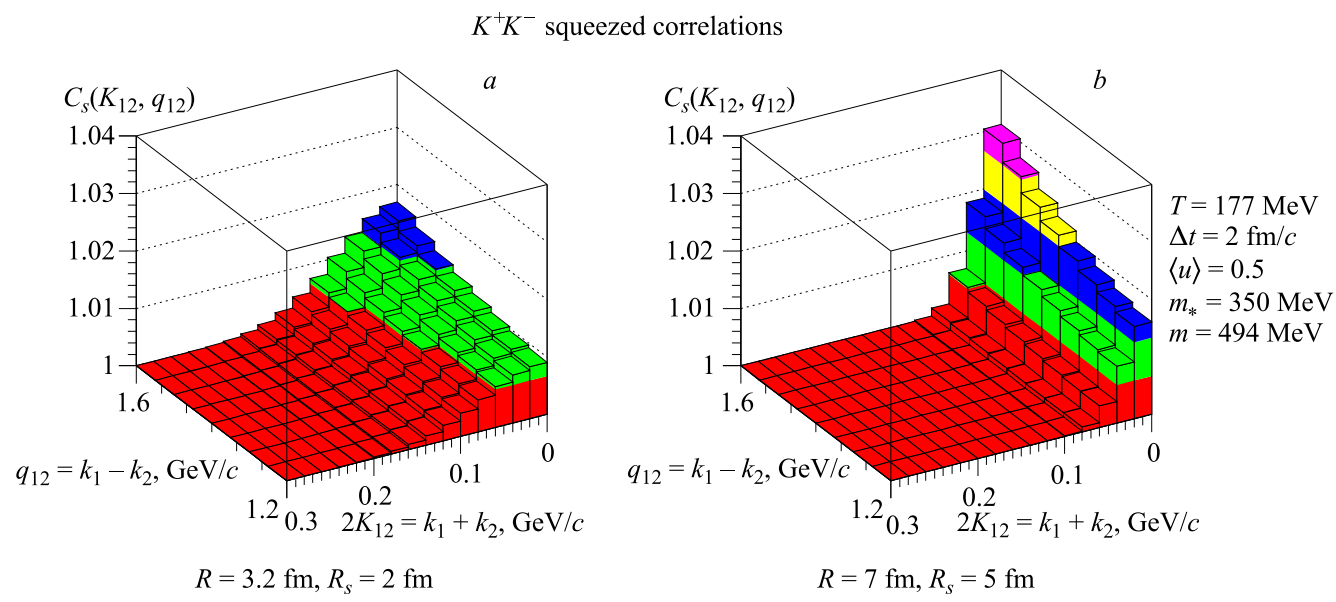

Fig. 3. $C\left(K_{12}, q_{12}\right)$ versus $K_{12}$ and $q_{12}$, for two sets of parameters for two corresponding histograms in Fig. 2, $b$

not allow for unambiguously concluding in favor of in-medium mass modification at RHIC energies. Nevertheless, it does not discard it either! Higher statistics data are necessary to help turn this crucial point into a positive or negative answer, besides solving the ambiguity of the parameters describing the system. The analysis of higher statistics data on $C\left(K_{12}, q_{12}\right)$ in the plane $\left(K_{12}, q_{12}\right)$ could improve the distinction of different scenarios and, hopefully, allow for concluding in favor of in-medium mass modification already at RHIC energies. 
126 Padula S. S., Dudek D. M., Socolowski O., Jr.

S.S.P. gratefully acknowledges the support by CAPES for participating in the WPCF 2010. D. M. D. also thanks CAPES for the support during the completion of this work.

\section{REFERENCES}

1. Andreev I. V., Plümer M., Weiner R. // Phys. Rev. Lett. 1991. V.67. P. 3475.

2. Asakawa M., Csörgö T., Gyulassy M. // Phys. Rev. Lett. 1999. V. 83. P. 4013.

3. Panda P. K. et al. // Phys. Lett. B. 2001. V.512. P. 49.

4. Padula S. S. et al. // Phys. Rev. C. 2006. V.73. P. 044906.

5. Dudek D. M., Padula S. S. // Phys. Rev. C. 2010. V.82. P. 034905.

6. Padula S. S., Socolowski O., Jr. // Phys. Rev. C. 2010. V. 82. P. 034908.

7. Csanád M. (for PHENIX Collab.) // Proc. of «Quark Matter 2005». Nucl. Phys. A. 2006. V. 774. P. 611.

8. Padula S. S. et al. // Proc. of «Quark Matter 2008». J. Phys. G.: Nucl. Part. Phys. 2008. V.35. P. 104141.

9. Nagy M. (for PHENIX Collab.) // WPCF 2009. http://indico.cern.ch/contributionDisplay.py?sessionId= $7 \&$ contribId=26\& confId $=54173$ 